\title{
Developing and Disseminating Data Analysis Tools for Open Science
}

Atsushi Mizumoto (Kansai University, Japan)

\section{Introduction}

Open science involves not only the products and outputs of our research but also the data analysis tools used throughout the process. Regardless of whether the research is quantitative or qualitative, in order to maximize the benefits of an open science approach, tools that facilitate data sharing and that reproduce the analysis with code should be adopted whenever possible. Table 1 illustrates open science research practices across the research cycle proposed in the tutorial "Easing into open science: A guide for graduate students and their advisors” (Kathawalla et al., 2021).

\section{Table 1}

Open Science Research Practices across the Research Cycle

(Adapted from Kathawalla et al., 2021)

\begin{tabular}{ll}
\hline Process & Practice \\
\hline 1. Conceptualization & $\begin{array}{l}\text { - Journal club } \\
\text { - Project workflow }\end{array}$ \\
\hline \multirow{2}{*}{ 2. Design } & - Preregistration \\
& - Registered reports \\
& - Data sharing planning \\
\hline 3. Analysis & - Reproducible code \\
\hline 4. Reporting & - Transparent writing \\
\hline 5. Dissemination & - Preprints \\
\hline
\end{tabular}


As can be seen in Table 1 (especially in the underlined practices), data sharing and reproducible code are at the core of the recommended open science practices. Hence, the tools must be chosen after careful consideration right from the beginning and at all stages of the research cycle.

According to the National Academies of Sciences, Engineering, and Medicine (2019), "reproducibility is obtaining consistent results using the same input data; computational steps, methods, and code; and conditions of analysis" (p. 6). It should be noted that, "replicability," sometimes used interchangeably with reproducibility, is "obtaining consistent results across studies aimed at answering the same scientific question, each of which has obtained its own data" (p. 6). Thus, reproducibility is a prerequisite for conducting a replication because unreproducible results cannot be replicated in another research setting. Robust, credible, and transparent high-quality research is only possible when reproducibility is guaranteed (Gass et al., 2020, p. 252). For these reasons, data analysis tools are essential for ensuring reproducibility.

At the same time, for science to be truly open, a wide range of high functionality and user-friendly tools need to be made freely available. In this chapter, I will first introduce publicly accessible tools that are often employed and cited in second language (L2) research or can be of potential interest to readers. Next, I will suggest some considerations to keep in mind when developing, deploying, and disseminating data analysis tools tailored for open science practices. The topics covered in this chapter are not only for software developers but also for researchers who intend to engage in reproducible research and open science practices. 


\section{Tools of Good Practice}

Table 2 shows some of the exemplary data analysis tools recommended for open science research practices. While the list is not comprehensive, it shows that the researchers in our field, or those in related fields, are willing to provide useful tools for free, driven by the purely altruistic reason of making them accessible to everyone.

In Table 2, the tools listed in the statistics category are all R-based computer programs. Luke Plonsky and $\mathrm{I}$ proposed that $\mathrm{R}$, an open-source programming language and environment (https://www.r-project.org/), should be the lingua franca (communication tool) for applied linguists (Mizumoto \& Plonsky, 2016). One of the most compelling advantages of using $\mathrm{R}$ is the reproducibility of data analysis that it enables. Using the raw data and the R code, it is possible for anyone to verify the computational reproducibility of the data analysis. Since reproducibility has gained attention over the years, many researchers have begun appreciating the advantages of using $\mathrm{R}$ in their open science research practices, which has been highlighted in other chapters in this volume (e.g., Huensch, this volume; Isbell, this volume). 
Table 2

Recommended Tools for Open Science Research Practices

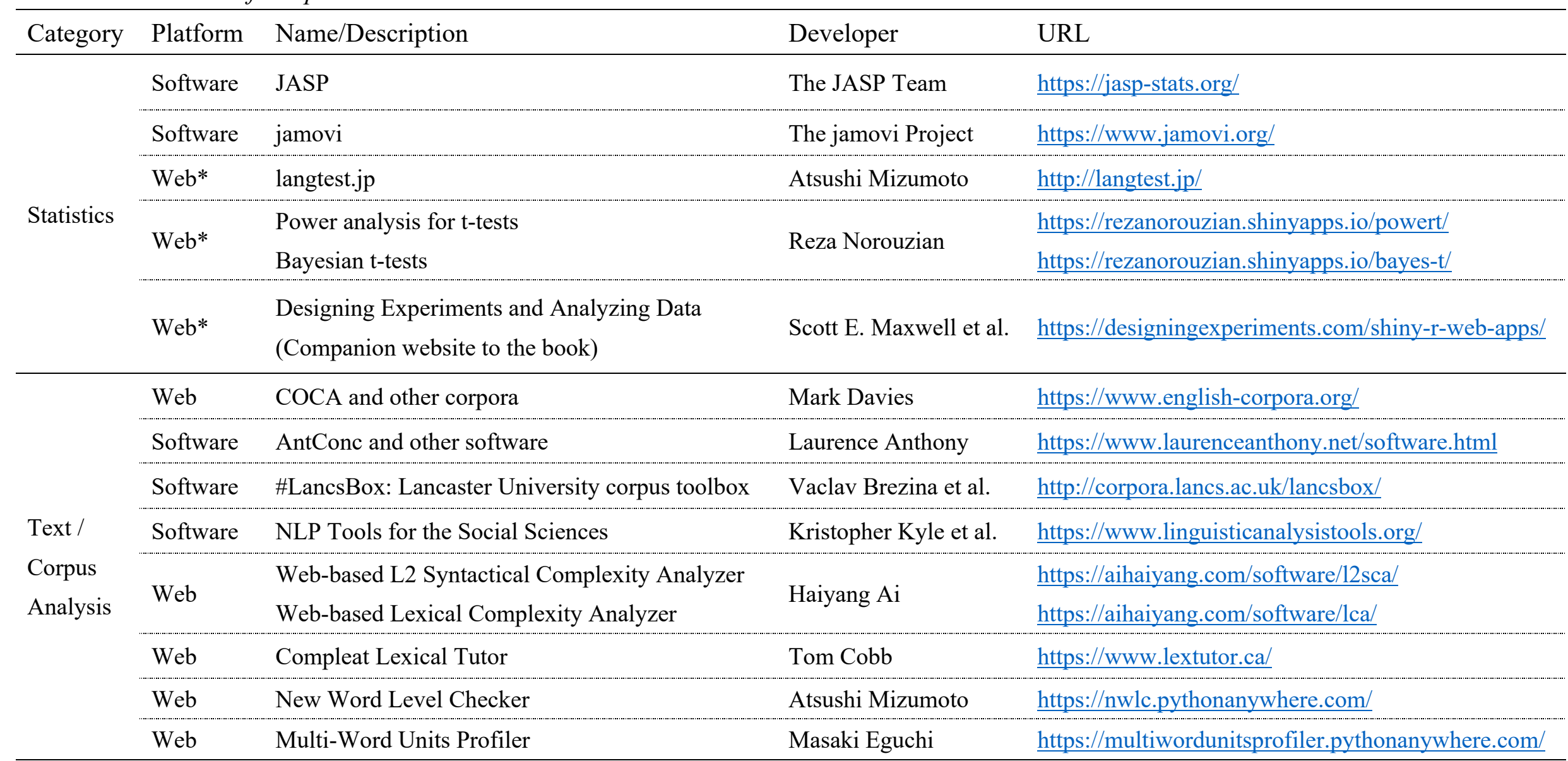

Note. Web*: Apps created using R Shiny. All software listed in the table are cross-platform (i.e., run on Windows, Mac, or Linux). 
The first two software packages (JASP and jamovi) have a click-and-point interface, a user-friendly feature for novice researchers. Both of these packages were developed as a viable, free alternative to IBM SPSS Statistics by the same initial development team, but are now developed and maintained by individuals with different philosophies in mind (Edelsbrunner, 2017). JASP focuses heavily on Bayesian statistics (Norouzian et al., 2019), and users can run a range of analyses using a Bayesian approach. In other words, JASP makes Bayesian analysis accessible to a broader audience. The disadvantage of JASP is that it emphasizes simplicity with a click-and-point interface and does not leave any analysis code behind. The development team has been working on adding that feature over the years (https://jasp-stats.org/faq/jasp-uses-r-analyses-possible-export-r-code-used-analysis/).

In contrast, jamovi produces the $\mathrm{R}$ code for each analysis in syntax mode. Although the number of analyses performed using jamovi is limited when compared with JASP, it is apparently superior to JASP in terms of reproducibility of data analysis. Furthermore, the jmv R package (Selker et al., 2021) makes it possible for users to execute all the analyses included in jamovi on R. In addition, although the number of analyses performed using jamovi is fewer, Bayesian statistics can be performed by adding the "jsq - Bayesian methods" module. As JASP and jamovi are both being developed daily, we should monitor them continuously as either of them could add a feature that can be of great value in reproducible data analysis.

The next three web applications are examples of how relatively easy it is to develop 
applications using the R Shiny package (https://shiny.rstudio.com/). While such programs are free, allowing anyone to perform various analyses online, the disadvantage is that, like any point-and-click software, it does not provide the analysis code. For this reason, users will not be able to reproduce the results unless they keep track of the raw data used and all the other settings in the analysis.

The next category in Table 2 is text/corpus analysis. Notably, the number of L2 studies utilizing corpora has expanded exponentially because of freely accessible web corpora (e.g., COCA) and software (e.g., AntConc and \#LancsBox), among other tools (see "Tools for Corpus Linguistics" (https://corpus-analysis.com/)). Additional tools included in Table 2 are software (natural language processing (NLP) tools for the social sciences) or web application (web-based L2 syntactical complexity analyzer and web-based lexical complexity analyzer), an online equivalent of Python-based L2 syntactical complexity analyzer (Lu, 2010) and lexical complexity analyzer (Lu, 2012). These tools provide, in a user-friendly manner, a wide variety of lexical and syntactic complexity metrics obtained using NLP approaches. The last three web applications are used for profiling vocabulary and multi-word units according to a selected list. These tools allow users to use a word list or a phrase list generated from large-scale corpora and analyze English texts, and are hence, examples of much-needful tools in the form of web applications.

One caveat with these text/corpus analysis tools is that, regardless of whether they are web applications or software, they do not produce the code used in the analysis in a way that facilitates 
reproduction of the analysis (e.g., like R). Worse yet, the raw data of the corpus (i.e., original texts) are often not accessible and cannot be made available because of copyright issues. Thus, when using these tools, users always need to take detailed notes of their analytic procedures and document them to be described in subsequent reports and for later use in a reproducible manner.

Although some tools listed in Table 2 still have room for improvement in terms of ensuring reproducibility (i.e., producing the code used in the analysis for later use), these tools are free and open to the community for anyone to use. If more and more researchers take similar initiatives, the transition to open science will be further facilitated and promoted. In the next section, I will discuss recommendations to be made when developing and disseminating tools to enhance reproducibility and open science.

\section{Recommendations for Developing and Disseminating Tools}

\section{- The tool should be provided free of charge.}

This is the first minimum criterion. All the tools in Table 2 are freely available. Considering the goals and principles of open science, it is a prerequisite that everyone should be able to use the tool free of charge. Once the developer starts charging users, the number of users inevitably drops, which impedes the spread of open science practices and, hence, the development of knowledge in the field. Additionally, if you were the developer, you would not want to charge your graduate students for accessing the tool. Therefore, bona fide researchers, not solely commercial entities, should be 
actively involved in developing data analysis tools.

- Code should be accessible to users.

As mentioned in the previous section, code produced using a certain analysis tool must be reproducible. In other words, code should be reusable so that researchers can produce the same result or inspect it. However, since it is often not possible to output the code used in all analyses, developers of the tool should publish the code on a code repository system, such as GitHub (https://github.com/), which is a source code version control platform. For example, Kristopher Kyle provided the Python code for the Tool for the Automatic Analysis of Lexical Diversity (TAALED) on GitHub (https://github.com/kristopherkyle/TAALED). If a code can be used by other researchers, many additional tools may be created by building on it. That is, it is a valuable way of repaying the research community we work in.

\section{- Documentation of the tool should be provided.}

When it comes to documentation (e.g., a user guide) of the tool, the degree of details included in it can vary from tool to tool. For users to check the compatibility of their computers with the tool (in the case of software on PC), information such as libraries and dependencies used in the versions to make them work should be preferably included. Nevertheless, the documentation should be easy to read and understand so that users can run the tool and replicate the examples given in the 
documentation. Documentation should be provided on the same website where the tools can be downloaded from or accessed. R Markdown (https://rmarkdown.rstudio.com/) can be helpful in producing such documents because it is fully reproducible (see example of R markdown in Liu \& De Cat, this volume). In addition to documentation, video tutorials may be helpful for some users. The video tutorials of AntConc (Anthony, 2020) on YouTube are a good example (https://youtube.com/playlist?list=PLiRIDpYmiC0Ta0-Hdvc1D7hG6dmiS_TZj).

Simultaneously, developers should keep in mind is that, in general, users do not read documentation (Novick \& Ward, 2006). Thus, developers should do their best to make the interface of the tool as intuitive as possible. Developers might also consider building help functions into the tool's interface.

\section{- Developers should aim for utmost accuracy.}

The developers of a tool should try their best to make the tool bug-free. As data analysis tools are the key to reproducible research practice, there should not be significant bugs in the program; the results must be scientifically accurate. In the first phase of development, tool developers should perform basic testing and validate the results by referring to similar programs. If possible, the code should be reviewed and tested by others to detect bugs and other problems before the official release. Developers can also invite colleagues, collaborators, and graduate students to "test drive" their tool as a means to identify flaws, glitches, inconsistencies, and so forth. 
- The tool should be cross-platform.

Limiting the operating system (OS), just like charging for a tool, limits the users and, as a result, does not maximally contribute to open science. Therefore, when developing software programs that run locally on the OS, developers should make the tool cross-platform (i.e., the tool must run on Windows, Mac, or Linux). Going cross-platform comes with its own set of hardships; developers must ensure that their tools work well with OS updates, whether Mac or Windows, and fix or modify them if necessary. Web applications do not have such problems, but they need to be checked and maintained regularly to ensure that they do not stop working when web browsers are updated. Developers of web-based tools may also want to verify that their tools maintain functionality when being used in tablets and other types of mobile devices.

\section{- The code or tool should be provided in a container format.}

To address the above-mentioned difficulties and challenges and to ensure reproducibility, tool developers should consider "containerization" of code, data, configuration, and computer architecture (e.g., OS) to reproduce exactly the same computing environments, irrespective of the user's OS (Harrison et al., 2021). There are many containerization tools, such as Docker (https://www.docker.com/), one of the most popular container services. Another container service, Code Ocean (https://codeocean.com/), integrates the code, data, environment, and dependencies into 
an online platform called "a compute capsule." Here is an example of a compute capsule I published for illustration (https://codeocean.com/capsule/2916503/tree). An open, robust research platform ecosystem, such as Code Ocean, is a great asset not only for developers but also for researchers who intend to share their code and data in a reproducible manner. This is because uploading data and code to a platform such as OSF (https://osf.io/) does not guarantee reproducibility as the software version, versions of libraries or packages, their dependencies, and the OS version will affect the success or failure in the process of reproducing the data analysis results (e.g., In'nami, Koizumi, Plonsky, \& Mizumoto, under review).

\section{- Developers should reach out to as many potential users as possible.}

Once a tool is released, it is advisable that developers write a paper describing the tool and publish it in a journal or in another outlet. This will increase the credibility and visibility of the tool, and users can cite the tool properly. Holding a workshop independently or at a conference will also be an effective way to disseminate the tool and ensure proper usage. In addition, social media where researchers collect information and exchange ideas may be a good place to start promoting the developed tool. For instance, the Facebook group "Applied Linguistics Research Methods Discussion" hosted by Luke Plonsky (https://www.facebook.com/groups/appliedlinguisticsresearchmethods) has over 23,000 individuals as of December 2021; thus, this is obviously where developers and users alike could spread the word 
about tools as well as share tips and suggestion for using them.

\section{Conclusion}

In this short chapter, I emphasized how data analysis tools play a vital role in the open science research cycle. I also introduced freely available tools that can be used for open science research practices in applied linguistics. Recommendations were given on what developers should keep in mind when developing, releasing, and disseminating their tools.

I hope that some readers are interested in and encouraged to develop their own useful tools. Developing tools is not exclusive to programmers. By learning how to develop tools and make them available, or at least sharing the data and code with everyone, one can truly contribute to the research community. Now, it is your turn to take action.

\section{References}

Anthony, L. (2020). AntConc (Version 3.5.9). https://www.laurenceanthony.net/software/antconc/ Edelsbrunner, P. (2017, March 23). Introducing jamovi: Free and open statistical software combining ease of use with the power of R. JEPS BULLETIN: The Official Blog of the Journal of European Psychology Students.

http://blog.efpsa.org/2017/03/23/introducing-jamovi-free-and-open-statistical-software-combi ning-ease-of-use-with-the-power-of-r/ 
Gass, S., Loewen, S., \& Plonsky, L. (2020). Coming of age: The past, present, and future of quantitative SLA research. Language Teaching, 54(2), 1-14. https://doi.org/10.1017/S0261444819000430

Harrison, S., Dasgupta, A., Waldman, S., Henderson, A., \& Lovell, C. (2021). How reproducible should research software be? Zenodo. https://doi.org/10.5281/zenodo.4761867

In’nami, Y., Koizumi, R., Plonsky, L., \& Mizumoto, A. (under review). Promoting reproducible research in applied linguistics. [Manuscript submitted for publication].

Kathawalla, U.-K., Silverstein, P., \& Syed, M. (2021). Easing into open science: A guide for graduate students and their advisors. Collabra: Psychology, 7(1), 18684. https://doi.org/10.1525/collabra.18684

Lu, X. (2010). Automatic analysis of syntactic complexity in second language writing. International Journal of Corpus Linguistics, 15(4), 474-496. https://doi.org/10.1075/ijcl.15.4.02lu

Lu, X. (2012). The relationship of lexical richness to the quality of ESL learners' oral narratives. The Modern Language Journal, 96(2), 190-208. https://doi.org/10.1111/j.1540-4781.2011.01232_1.x

Mizumoto, A., \& Plonsky, L. (2016). R as a lingua franca: Advantages of using R for quantitative research in applied linguistics. Applied Linguistics, 37(2), 284-291. https://doi.org/10.1093/applin/amv025

National Academies of Sciences, Engineering, and Medicine. (2019). Reproducibility and 
replicability in science (p. 25303). National Academies Press. https://doi.org/10.17226/25303

Norouzian, R., Miranda, M. D., \& Plonsky, L. (2019). A Bayesian approach to measuring evidence in L2 research: An empirical investigation. The Modern Language Journal, 103(1), 248-261. https://doi.org/10.1111/modl.12543

Novick, D. G., \& Ward, K. (2006). What users say they want in documentation. Proceedings of the 24th Annual Conference on Design of Communication, 84.

https://doi.org/10.1145/1166324.1166346

Selker, R., Love, J., Dropmann, D., \& Moreno, V. (2021). jmv: The “jamovi” analyses. https://CRAN.R-project.org/package=jmv 